\title{
Sense-antisense gene overlap is probably a cause for retaining the few introns in Giardia genome and the implications
}

\author{
Min Xue ${ }^{1,2}$, Bing Chen ${ }^{1}$, Qingqing Ye ${ }^{1}$, Jingru Shao ${ }^{1}$, Zhangxia Lyu ${ }^{1}$ and Jianfan Wen ${ }^{1 *}$
}

\begin{abstract}
Background: It is widely accepted that the last eukaryotic common ancestor and early eukaryotes were intron-rich and intron loss dominated subsequent evolution, thus the presence of only very few introns in some modern eukaryotes must be the consequence of massive loss. But it is striking that few eukaryotes were found to have completely lost introns. Despite extensive research, the causes of massive intron losses remain elusive. Actually the reverse question -how the few introns can be retained under the evolutionary selection pressure of intron loss -- is equally significant but was rarely studied, except that it was conjectured that the essential functions of some introns prevent their loss. The situation that extremely few (eight) spliceosome-mediated cis-spliced introns present in the relatively simple genome of Giardia lamblia provides an excellent opportunity to explore this question.

Results: Our investigation found three types of distribution patterns of the few introns in the intron-containing genes: ancient intron in ancient gene, later-evolved intron in ancient gene, and later-evolved intron in later-evolved gene, which can reflect to some extent the dynamic evolution of introns in Giardia. Without finding any special features or functional importance of these introns responsible for their retention, we noticed and experimentally verified that some introncontaining genes form sense-antisense gene pairs with transcribable genes on their complementary strands, and that the introns just reside in the overlapping regions.
\end{abstract}

Conclusions: In Giardia's evolution, despite constant evolutionary selection pressure of intron loss, intron gain can still occur in both ancient and later-evolved genes, but only a few introns are retained; at least the evolutionary retention of some of the introns might not be due to the functional constraint of the introns themselves but the causes outside of introns, such as the constraints imposed by other genomic functional elements overlapping with the introns. These findings can not only provide some clues to find new genomic functional elements -- in the areas overlapping with introns, but suggest that "functional constraint" of introns may not be necessarily directly associated with intron loss and gain, and that the real functions are probably still outside of our current knowledge.

Reviewers: This article was reviewed by Mikhail Gelfand, Michael Gray, and Igor Rogozin.

Keywords: Evolutionary retention of introns, Gene overlap, Intron evolution, Genome evolution, Giardia lamblia

\footnotetext{
* Correspondence: wenjf@mail.kiz.ac.cn

'State Key Laboratory of Genetic Resources and Evolution, Kunming Institute of Zoology, Chinese Academy of Sciences, Kunming 650223, Yunnan, China Full list of author information is available at the end of the article
}

(c) The Author(s). 2018 Open Access This article is distributed under the terms of the Creative Commons Attribution 4.0 International License (http://creativecommons.org/licenses/by/4.0/), which permits unrestricted use, distribution, and reproduction in any medium, provided you give appropriate credit to the original author(s) and the source, provide a link to the Creative Commons license, and indicate if changes were made. The Creative Commons Public Domain Dedication waiver (http://creativecommons.org/publicdomain/zero/1.0/) applies to the data made available in this article, unless otherwise stated. 


\section{Background}

Spliceosomal introns are a common feature of all eukaryotic nuclear genomes, but their number and density in a genome vary dramatically among different species [1, 2], ranging from less than 0.5 intron/gene in some protists such as the Microsporidian Encephalitozoon species [3] and Cyanidioschyzon merolae [4] to over 18 per gene in Symbiodinium minutum [5] (even larger than those of most mammals, which is generally over eight/gene [6]). Accumulating evidence suggests that the last eukaryotic common ancestor (LECA) and early eukaryotes were relatively intron rich, with subsequent genome evolution dominated by intron loss, and thus those contemporary eukaryotes with remarkably few introns must have experienced massive intron loss secondarily [7-9]. But, interestingly, almost no eukaryotes with sequenced genomes were found to have completely lost their introns so far, except two Microsporidia, Nematocida parisii and Nematocida sp1 [10].

Unfortunately, why introns were lost, especially massively lost in some eukaryotes, remains obscure despite extensive research $[11,12]$. Actually, the reverse question -how introns, especially the few ones in intron-poor eukaryotes, can be retained under the evolutionary selection pressure of intron loss -- is equally important, but was rarely carefully studied. Although some authors thought that the reason for the retention of introns in genomes is likely the essential functions of these introns [13, 14], such as some introns can be functioning as important non-coding DNA sequences, and regulate the levels of mRNA transcription, processing, transport and so on $[15,16]$, the 'functional constraint' scenario -- only the introns with important functions can get rid of the fate of being lost -- lacks evidence that the lost introns are all useless or less useful than the retained ones in any eukaryotes, and moreover, actually the functions of introns are still far from being well understood [17]. Therefore, the investigation of the evolutionary retention of intron might be helpful not only to answering the question about intron loss but also to understanding the function and evolution of introns.

Giardia lamblia is a parasitic protozoan belonging to Diplomonadida (Excavata). It has a very minimalistic genome, compact in structure and content [18], and only eight spliceosomal introns were found in its genome [18-23]. Thus it can be speculated that this organism must have undergone massive intron loss, with only very few left in the genome. Therefore, this organism may provide an excellent opportunity for exploring how the few introns were retained. In the present work, by investigating the intron-containing genes and the few introns of Giardia, besides finding the distribution patterns that can reflect the dynamic evolution of intron to some extent in Giardia, we observed and experimentally confirmed an interesting phenomenon that sense-antisense
(SAS) gene overlaps appear in the areas of some introns, and thus "overlap constraint" might be at least one of the causes for preventing introns from being lost, though it is uncertain whether the other retained introns also overlap with any unknown genomic functional elements yet. The implications of these findings for intron evolution and function are discussed.

\section{Results \\ Characteristics of the intron-containing genes and their introns in Giardia genome}

In the genome database of G. lamblia, GiardiaDB, four of the eight Giardia intron-containing genes are annotated to code proteins homlogous to known proteins in common eukaryotes, and the other four to code hypothetical proteins (Table 1). Our investigation (mainly by sequence comparative analysis) indicated that: 1) the former four intron-containing genes are all common eukaryotic-conserved genes, which are thus most likely vertically inherited from the common ancestor of these eukaryotes and are very 'ancient', while the latter four are all Giardia-specific genes (not found in any other eukaryotes including other Excavata species), which thus most likely emerged after the divergence of Giardia from other Excavata and are 'later-evolved' genes compared with the former ones; 2) the introns in the three (GL50803-15604, GL50803-15124, GL50803-17244) of the four ancient genes are eukaryotic-conserved (Additional file 1), and thus they are 'ancient' introns in ancient genes, while the intron in the other one ancient gene (GL50803-27266) is a Giardia-specific intron (not found in other eukaryotes including other Excavata species), and thus this intron most likely emerged after the divergence of Giardia from other Excavata and is a 'later-evolved' intron in an ancient gene; 3) all the four Giardia-specific ('later-evolved') intron-containing genes (GL50803-37070, GL5080335332, GL50803-15525 and GL50803-86945), which account for only about $0.6 \%$ of all the $\sim 700$ Giardia-specific protein-coding genes in the genome [18], each contain an Giardia-specific intron (not found in other eukaryotes including other Excavata species), and thus the four introns all are 'later-evolved' introns in 'later-evolved' genes (Table 1).

These observations suggest that: 1) while Giardia massively lost its introns, new introns also arose in both ancient and later-evolved genes; 2) the evolutionary selection pressure of intron loss seems to constantly exist in the whole evolutionary process of Giardia, but only a few of both the ancient and later-evolved introns have been retained in the genome.

To find the reason why these few introns can be retained in Giardia genome under the constant evolutionary selection pressure of loss, we investigated the 
Table 1 The integrated information of the eight spliceosome-mediated introns and their host genes and complementary strands

\begin{tabular}{|c|c|c|c|c|c|}
\hline \multicolumn{3}{|c|}{ Intron-containing gene } & \multicolumn{2}{|l|}{ Intron } & \multirow[t]{2}{*}{ Complementary strand } \\
\hline Gene ID & Product & Age & Age & Size (bp) & \\
\hline GL50803-27266 & $2 \mathrm{Fe}-2 \mathrm{~S}$ ferredoxin & ancient & later evolved & 35 & No ORF, no transcripts detected \\
\hline GL50803-15604 & 265 proteasome non-ATPase regulatory subunit 4 & & ancient & 29 & No ORF, no transcripts detected \\
\hline GL50803-15124 & Dynein light chain & & & 32 & No ORF, no transcripts detected \\
\hline GL50803-17244 & Ribosomal Protein L7A & & & 109 & Antisense gene with transcripts \\
\hline GL50803-37070 & Hypothetical protein & later evolved & later evolved & 41 & Antisense gene with transcripts \\
\hline GL50803-35332 & Hypothetical protein & & & 220 & No ORF, no transcripts detected \\
\hline GL50803-15525 & Hypothetical protein & & & 33 & No ORF, no transcripts detected \\
\hline GL50803-86945 & Hypothetical protein & & & 36 & No ORF, no transcripts detected \\
\hline
\end{tabular}

These two SAS gene overlaps are highlighted in boldface

characteristics of these introns in many aspects. It had been shown that seven of the eight Giardia introns are bounded by canonical GT-AG splice signals, only one, the [2Fe-2S] ferredoxin (GL50803-27266) intron, has a noncanonical splice signal CT-AG [20]. Most of the eight introns are small (most of them are less than $40 \mathrm{bp}$ long and their numbers of nucleotide are not the multiple of three) and do not have any conserved sequence motifs. Our further analysis predicted no special secondary structures that would be able to form in these introns. Besides, our survey also showed that there were not any reports about alternative splicing of the two introns in genes GL5080315525 and GL50803-86945 [19, 23].

Interestingly, on the complementary strands, we found that two intron-containing genes, GL50803-17244 (ribosomal protein L7a gene) and GL50803-37070 (a "hypothetical protein" gene), each have an antisense gene, GL50803-20429 and GL50803-28204, which are just annotated as "hypothetical protein" and "unspecified product" in the genome database, respectively. That is, the two intron-containing genes and their antisense gene form SAS gene pairs. We thought this phenomenon might be related to the intron retention. Nevertheless, the two anti-sense genes need to be further verified, and the details of the overlaps with their sense genes also need to be analyzed in detail, both of which are carried out at below.

\section{Verification of the antisense genes}

The strand-specific RT-PCR of the two antisense genes, GL50803-28204 and GL50803-20429, generated two products with the expected lengths of 172 and $288 \mathrm{bp}$, respectively. The sequencing further confirmed that the two products just seem to be transcribed from the opposite direction of the two sense (intron-containing) genes, GL50803-37070 and GL50803-17244, respectively, and the two introns are just located within the two overlapping regions of the two SAS gene pairs, respectively (Fig. 1).
The RACE of the two antisense genes generated a 1232 bp product for GL50803-28204 and a 1177 bp product for GL50803-20429. After sequencing and comparing with their corresponding genomic DNA sequences in the GiardiaDB database, we found the two antisense genes contain no introns, especially in the regions corresponding to the two introns of the sense genes. But the software ORF Finder predicted that the largest ORFs of the two antisense genes are only 264 bp for GL50803-28204 and 363 bp for GL50803-20429, and moreover, no proteins homologous to the putative proteins coded by the two largest ORFs could be found in other organisms including Giardia's close relative Spironucleus in GenBank.

\section{Discussion}

When investigating the characteristics of the eight intron-containing genes and their corresponding introns in Giardia genome, we found the evolutionary selection pressure of intron loss seems to constantly exist in the whole evolutionary course of Giardia, but a few introns have been retained in modern Giardia. However, we did not find any special regularities or unusualnesses responsible for the intron retention. This suggests that there may be neither any special structural features nor necessarily any functional importance of introns responsible for the intron retention, though many cases of how introns exert their influences on eukaryotic gene expression were reported $[15,16]$. This is consistent with the fact that so many introns, at least part of which definitely possesses important functions, have been lost in intron-poor eukaryotes like Giardia. Thus, the reasons for the retention might lie outside the intron-containing genes and the introns themselves.

Interestingly, we noticed that on the complementary strands of two of the eight intron-containing genes, GL50803-37070 and GL50803-17244, there exist correspondingly two anti-sense genes, GL50803-28204 and GL50803-20429. By strand-specific RT-PCR, RACE and sequencing, we got the transcripts and sequences of the 


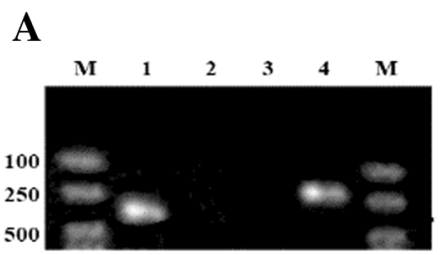

C

GL50803-20429:

AAAGGTGGGCTTGTCCTCTGGGTTCACGTCGGTGAAGCAGACGCTGGTCGTCTTCTTC AGATGGACGAGCTTGCCCAGATCGCCCTTA GTGCGAACGATGGCGTACGGGACGCCC ATCTTGTGACAGAGTGTGGGAAGCCAAAGTACGAGCTGTGGGTCAGTTGTCAGGTGA ACAGCGAAGTCCACCCGCTGACAACACACAACCCGCAATCAGAGGTGTGTGCGGTCAG CGGACGGCTCCTCGCGCATAAGAACATACTTCAAGGGGGTCGACATCATTTGCAATCA

\section{D}

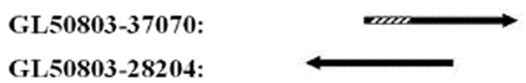

GL50803-17244:

GL50803-20429:

Fig. 1 Results of strand-specific RT-PCR and sequencing of the two antisense genes and their corresponding schematic diagram. a Lane 1, Strand-specific RT-PCR product of the GL50803-20429; Lane 4, the Strand-specific RT-PCR product of the GL50803-28204; Lane 2 and lane 3, negative controls (with no RTase) corresponding to lane 1 and lane 4, respectively; M, molecular markers. b Nucleotide sequence of GL5080328204 gene acquired by Strand-specific RT-PCR and sequencing. The locations of the primers are underlined. c Nucleotide sequence of GL5080320429 gene acquired by Strand- specific RT-PCR and sequencing. $\mathbf{d}$ Schematic diagram of the two SAS gene pairs. The sequence lengths of GL50803-28204 and GL50803-20429 are according to the Strand-specific RT-PCR products, and the lengths of GL50803-37070 and GL5080317244 are based on the GiardiaDB database. Arrow represents the orientation of transcription; and the dashed box and solid lines represent introns and exons, respectively

two genes, and found they both have no introns. Thus the two anti-sense genes have been verified to be really genes that are actively transcribed. And actually the anti-sense gene GL50803-20429 has been reported to be a mRNA gene being expressed during excystation and encystation, and in trophozoites but not cysts [24]. As for the other anti-sense gene GL50803-28204, it has a quite short putative ORF but has no homologs in other organisms including Giardia's close relative Spironucleus. Besides, the total RNA had been processed using Poly(A) Polymerase to add a poly(A) tail at the 3 'ends before we performed rapid amplification of their cDNA 3'ends, thus from the experiment we still do not know whether the transcripts of GL50803-28204 are polyadenylated or not, namely, it is unclear whether the transcripts of GL50803-28204 are mRNA or not. Therefore, we can only conjecture that GL50803-28204 is either a non-protein-coding or Giardia-specific protein-coding gene. Considering that many antisense genes overlapped with protein-coding genes are non-coding RNA genes in Giardia [25], the antisense gene identified here might also be noncoding RNA gene. But there is still no tangible evidence for what the two genes are despite our lots of experimental efforts (not shown) to identify them.
Whatever the antisense genes code for, our work showed that they are transcribable genes and form SAS gene overlap with their intron-containing sense genes, and that the introns just reside in the overlap regions. Considering that the gene sequence mutation (especially deletion) cannot occur randomly, the antisense genes must have imposed the restriction of variation (especially deletion) on the introns of the sense genes in the overlapping areas, and thus such a kind of SAS gene overlap must have prevented the introns from being lost.

We have also analyzed the corresponding genome sequences of the two anti-sense genes in the other four Giardia isolates with genome sequenced, and found that the two anti-sense genes (exactly corresponding to GL50803-28204 and GL50803-20429 in isolate WB) also exist in isolate $\mathrm{DH}$, but not in the other three isolates. However, interestingly, other ORFs (not exactly corresponding to GL50803-28204) can be found on the complementary strands to overlap with the intron corresponding to the intron in the gene GL50803-37070 in P15, GS, GS-B (Additional file 2). Therefore, such SAS gene overlap is not just found in isolate $\mathrm{WB}$, but is at least a common phenomenon in other isolates of Giardia. 
As for the other six introns, we did not find any ORFs on their corresponding complementary strands. Although we also experimentally examined whether their complementary strands (especially the areas overlapping with these introns) are transcribed, no transcripts were found (Additional file 3). Nevertheless, it is uncertain whether the corresponding complementary strands of these introns are resided in by some unknown genomic functional elements which are not generally transcribed at all. If this is true, these introns are also retained by the same cause -- overlap constrain -- as the former two introns. Certainly, it is also possible that the six introns are retained by other unknown reasons. We have also analyzed the intron regions of many intron-poor eukaryotes including Microsporidia, Trichomonas, Spironucleus, but unfortunately did not find such sense-antisense gene overlaps as in Giardia (data not shown). But considering that some genes overlap with the UTRs of the adjacent genes on the opposite strand (as found in Antonospora locustae and Encephalitozoon cuniculi [26]) and that UTR information of many genes is unavailable from current databases, and thus such overlapping regions are actually difficult to be found out currently. More importantly, some introns may overlap with some unknown non-coding and non-transcribable genomic functional elements, since so huge components of eukaryotic genomes are still undetermined. Therefore, the lack of information of genomes may be the main cause for that few SAS gene overlaps involving intron regions can be identified at present.

Theoretically, overlapping with any genomic functional elements on either the same strand or the complementary one (namely, either same-strand overlapping or different-strand overlapping) can result in intron retention, as long as the introns are just in the overlapping areas. Therefore, since such overlapping structures are widely distributed in eukaryotes [27], it can be expected that quite a number of introns in diverse eukaryotes may also be retained due to this kind of "overlap constraint". We believe that more and more examples might be able to be found in diverse eukaryotes in the future. Conversely, such an intron retention phenomenon probably can provide a valuable clue to find new genomic functional elements - in the overlapping area with introns.

\section{Conclusions}

In conclusion, by investigating the extremely intron-poor eukaryote Giardia, we have revealed some interesting findings about the dynamic evolution of introns in the intron-poor eukaryotes: the evolutionary selection pressure of intron loss may constantly exist in these eukaryotes, but new introns can still arise either in ancient genes or later-evolved genes, but only a few introns can be retained in the genome; the retention of the few introns may not be necessarily caused by functional constraint of the introns themselves but due to the reasons outside of the introns, and "overlap constraint" imposed by other genomic functional elements overlapping with the introns is at least one of such causes. Our finding may conversely provide a clue to find new genomic functional elements (which was probably traditionally regarded as "junk" and is still undetermined) in such kinds of overlap regions. Most importantly, our work implicates that "functional constraint" of introns is not necessarily directly associated with intron loss and gain, and even that the real functions or the way of functioning of introns are probably still outside of our current knowledge. Therefore this work may be able to shed some new lights on the research of evolution and function of introns and genomes.

\section{Methods}

\section{Database and bioinformatics methods}

The template sequences for designing primers of Giardia genes were downloaded from GiardiaDB (1 december 2017 release) [28]. The software ORF Finder were used to predict the ORFs of the RACE products (see blow), then the predicted ORFs were used as queries to search their homologs with Blastp against the NCBI non-redundant protein sequences (nr) database. The program RNAfold web server was used to analyze the secondary structure of introns. The sequences of the four genes and their coding proteins, $2 \mathrm{Fe}-2 \mathrm{~S}$ ferredoxin, $26 \mathrm{~S}$ proteasome non-ATPase regulatory subunit 4 , dynein light chain, and ribosomal protein L7A from other organisms were identified and collected by Blastp searching against GenBank with Giardia's corresponding sequences as queries. Protein alignments were generated with ClustalX 2 applying default alignment parameters. The introns in the genes were determined by comparing cDNA and gene sequences. The other four intron-containing genes with annotated as hypothetical protein also identified by sequence comparative analysis to determine whether they are Giardia-specific or not.

\section{Giardia cultures}

The cell line of WB isolate (assemblage A), namely WB clone C6 (ATCC 50803), was used in the study. Its cultures were routinely grown in filter-sterilized TYI-S-33 medium supplemented with bovine bile in glass screw cap tubes at $37^{\circ} \mathrm{C}$ and were sub-cultured every 3 to 4 days.

\section{RNA extraction}

Giardia total RNA was extracted and treated to remove any contaminated genomic DNA by RNAprep Pure Cell/Bacteria Kit (TIANGEN) using about $5 \times 10^{6}$ Giardia trophozoites that were harvested by ice-slush incubation and centrifuged at $6000 \mathrm{~g}$ for $5 \mathrm{~min}$ 
according to the manufacturer's instructions. The quality and quantity of the RNA preparation were assessed with agarose gel electrophoresis and the absorption at 260 and $280 \mathrm{~nm}$.

\section{Strand-specific RT-PCR}

First-strand cDNAs of the two antisense genes, GL50803-28204 and GL50803-20429, were synthesized from $500 \mathrm{ng}$ DNase-I treated total RNA per reaction at $54{ }^{\circ} \mathrm{C} 30 \mathrm{~min}, 99^{\circ} \mathrm{C} 5 \mathrm{~min}$, and $5{ }^{\circ} \mathrm{C} 5 \mathrm{~min}$ with the specific antisense primer $4 \mathrm{C}$ and $9 \mathrm{C}$ respectively instead of the reverse primers in the kit, and then amplified with specific primers pairs of $4 \mathrm{~A} / 4 \mathrm{~S}$ and $9 \mathrm{~A} / 9 \mathrm{~S}$ by using RNA PCR Kit (AMV) Ver.3.0(Takara, Japan). The PCR conditions were as follows: $94{ }^{\circ} \mathrm{C}$ for $30 \mathrm{~s}$, followed by $30 \mathrm{cy}-$ cles of $94{ }^{\circ} \mathrm{C}$ for $30 \mathrm{~s}, 55^{\circ} \mathrm{C}$ for $30 \mathrm{~s}$ and $72{ }^{\circ} \mathrm{C}$ for $45 \mathrm{~s}$. The PCR products were purified using the Wizard SV Gel and PCR Clean-Up System kit (Qiagen, Germany), and cloned into pMD-19 T vectors from $35 \mathrm{ng}$ purified PCR products using TaKaRa pMD-19 T Vector Cloning Kit (TaKaRa, Japan) according to the manufacturer's instructions. Then, the ligation products were transformed into DH5 $\alpha$ Chemically Competent E. coli. Colony PCR with vector-specific primers provided in the kit was adopted to select colonies. These selected colonies were sequenced using vector-specific forward and reverse primers by Sangon Biology Company (Shanghai, China).

\section{Rapid amplification of cDNA ends}

The total RNA were processed using Poly(A) Polymerase(TaKaRa, Japan) to add a poly(A) tail at the 3' ends of the RNA before performing rapid amplification of their cDNA 3'ends. We experimentally determined the 3 'ends by using nested PCR primer (3R4O/3R4I and 3R9O/3R9I) according to the RNA PCR Kit (AMV) Ver.3.0 (Takara, Japan). 5'-RACE was performed by using a SMARTer RACE 5'/3'Kit (TaKaRa, Japan) with $500 \mathrm{ng}$ total RNA as the template and the gene-specific 5'-RACE primers 5R4 and 5R9 for the two antisense genes, GL50803-28204 and GL50803-20429, according to the manufacturer's instructions. Both the 3'-RACE and 5'-RACE primers (Additional file 4) were designed based on the transcripts from the Strand-specific RT-PCR. The RACE-PCR products were analyzed by agarose gel electrophoresis and sequenced as described above. Please see the reviewers' comments in the Additional file 5.

\section{Additional files}

Additional file 1: The conservative analysis of Giardia's introns among diverse eukaryotes. (DOC 14 kb)
Additional file 2: The Analysis results of the two SAS gene pairs in Giardia isolates. (DOCX $51 \mathrm{~kb}$ )

Additional file 3: Results of strand-specific RT-PCR of the complementary areas of the other six introns of G. lamblia. (DOC $517 \mathrm{~kb}$ )

Additional file 4: The primers designed for the strand-specific RT-PCR and RACE of the complementary areas of the eight introns of G. lamblia. (DOC $79 \mathrm{~kb}$ )

Additional file 5: Reviewers' comments. (DOCX 20 kb)

\section{Abbreviations}

LECA: Last eukaryotic common ancestor; RACE: Rapid amplification of cDNA ends; SAS: Sense-antisense

\section{Funding}

This work was supported by the National Natural Science Foundation of China (NSFC) (grant numbers 31572256, 31772452, 31401972 and 31401973) and the Natural Science Foundation of Yunnan Province (grant number 2015FB181).

\section{Availability of data and materials}

All data generated or analysed during this study are included in this published article and its supplementary information files.

\section{Authors' contributions}

JW designed and supervised this study. MX performed genetic

characterization work. MX, BC, QY, JS, and ZL analyzed the data. MX and JW wrote the manuscript. All authors read and approved the final manuscript.

Ethics approval and consent to participate

Not applicable

\section{Consent for publication}

Not applicable

\section{Competing interests}

The authors declare that they have no competing interests.

\section{Publisher's Note}

Springer Nature remains neutral with regard to jurisdictional claims in published maps and institutional affiliations.

\section{Author details}

${ }^{1}$ State Key Laboratory of Genetic Resources and Evolution, Kunming Institute of Zoology, Chinese Academy of Sciences, Kunming 650223, Yunnan, China. ${ }^{2}$ Kunming College of Life Science, University of Chinese Academy of

Sciences, Kunming 650204, Yunnan, China.

Received: 21 June 2018 Accepted: 2 October 2018

Published online: 17 October 2018

\section{References}

1. Csuros M, Rogozin IB, Koonin EV. A detailed history of intron-rich eukaryotic ancestors inferred from a global survey of 100 complete genomes. PLoS Comput Biol. 2011;7(9):e1002150.

2. Roy SW. Intron-rich ancestors. Trends Genet. 2006;22(9):468-71.

3. Pombert JF, Selman M, Burki F, Bardell FT, Farinelli L, Solter LF, Whitman DW, Weiss LM, Corradi N, Keeling PJ. Gain and loss of multiple functionally related, horizontally transferred genes in the reduced genomes of two microsporidian parasites. Proc Natl Acad Sci U S A. 2012;109(31):12638-43.

4. Stark MR, Dunn EA, Dunn WS, Grisdale CJ, Daniele AR, Halstead MR, Fast NM, Rader SD. Dramatically reduced spliceosome in Cyanidioschyzon merolae. Proc Natl Acad Sci U S A. 2015;112(11):E1191-200.

5. Shoguchi E, Shinzato C, Kawashima T, Gyoja F, Mungpakdee S, Koyanagi R, Takeuchi T, Hisata K, Tanaka M, Fujiwara M, et al. Draft assembly of the Symbiodinium minutum nuclear genome reveals dinoflagellate gene structure. Curr Biol. 2013;23(15):1399-408.

6. Roy SW, Gilbert W. The evolution of spliceosomal introns: patterns, puzzles and progress. Nat Rev Genet. 2006;7(3):211-21. 
7. Rodriguez-Trelles F, Tarrio R, Ayala FJ. Origins and evolution of spliceosomal introns. Annu Rev Genet. 2006;40:47-76.

8. Irimia M, Roy SW. Origin of spliceosomal introns and alternative splicing. Cold Spring Harb Perspect Biol. 2014:6(6).

9. Koonin EV. Intron-dominated genomes of early ancestors of eukaryotes. J Hered. 2009;100(5):618-23.

10. Cuomo CA, Desjardins CA, Bakowski MA, Goldberg J, Ma AT, Becnel JJ, Didier ES, Fan L, Heiman DI, Levin JZ, et al. Microsporidian genome analysis reveals evolutionary strategies for obligate intracellular growth. Genome Res. 2012;22(12):2478-88.

11. Cohen NE, Shen R, Carmel L. The role of reverse transcriptase in intron gain and loss mechanisms. Mol Biol Evol. 2012;29(1):179-86.

12. Mitrovich QM, Tuch BB, De La Vega FM, Guthrie C, Johnson AD. Evolution of yeast noncoding RNAs reveals an alternative mechanism for widespread intron loss. Science. 2010;330(6005):838-41.

13. Roy SW, Penny D. Widespread intron loss suggests retrotransposon activity in ancient apicomplexans. Mol Biol Evol. 2007;24(9):1926-33.

14. Hooks KB, Delneri D, Griffiths-Jones S. Intron evolution in Saccharomycetaceae. Genome Biol Evol. 2014;6(9):2543-56.

15. Rogozin IB, Sverdlov AV, Babenko VN, Koonin EV. Analysis of evolution of exon-intron structure of eukaryotic genes. Brief Bioinform. 2005;6(2):118-34.

16. Rogozin IB, Carmel L, Csuros M, Koonin EV. Origin and evolution of spliceosomal introns. Biol Direct. 2012;7(1):11.

17. Carmel L, Wolf YI, Rogozin IB, Koonin EV. Three distinct modes of intron dynamics in the evolution of eukaryotes. Genome Res. 2007;17(7):1034-44.

18. Morrison HG, McArthur AG, Gillin FD, Aley SB, Adam RD, Olsen GJ, Best AA, Cande WZ, Chen F, Cipriano MJ, et al. Genomic minimalism in the early diverging intestinal parasite Giardia lamblia. Science. 2007;317(5846):1921-6.

19. Kamikawa R, Inagaki Y, Hashimoto T. Secondary loss of a cis-spliced intron during the divergence of Giardia intestinalis assemblages. BMC Res Notes. 2014;7:413.

20. Nixon JE, Wang A, Morrison HG, McArthur AG, Sogin ML, Loftus BJ, Samuelson J. A spliceosomal intron in Giardia lamblia. Proc Natl Acad Sci U S A. 2002;99(6): 3701-5.

21. Russell AG, Shutt TE, Watkins RF, Gray MW. An ancient spliceosomal intron in the ribosomal protein L7a gene (Rpl7a) of Giardia lamblia. BMC Evol Biol. 2005:5:45.

22. Roy SW, Hudson AJ, Joseph J, Yee J, Russell AG. Numerous fragmented spliceosomal introns, AT-AC splicing, and an unusual dynein gene expression pathway in Giardia lamblia. Mol Biol Evol. 2012;29(1):43-9.

23. Franzen O, Jerlstrom-Hultavist J, Einarsson E, Ankarklev J, Ferella M, Andersson B, Svard SG. Transcriptome profiling of Giardia intestinalis using strand-specific RNA-seq. PLoS Comput Biol. 2013;9(3):e1003000.

24. Birkeland SR, Preheim SP, Davids BJ, Cipriano MJ, Palm D, Reiner DS, Svard SG, Gillin FD, McArthur AG. Transcriptome analyses of the Giardia lamblia life cycle. Mol Biochem Parasitol. 2010;174(1):62-5.

25. Chen XS, White WT, Collins $\sqcup$, Penny D. Computational identification of four spliceosomal snRNAs from the deep-branching eukaryote Giardia intestinalis. PLoS One. 2008;3(8):e3106.

26. Corradi N, Gangaeva A, Keeling PJ. Comparative profiling of overlapping transcription in the compacted genomes of microsporidia Antonospora locustae and Encephalitozoon cuniculi. Genomics. 2008;91(4):388-93.

27. Kumar A. An overview of nested genes in eukaryotic genomes. Eukaryot Cell. 2009;8(9):1321-9.

28. Shabalina SA, Ogurtsov AY, Kondrashov VA, Kondrashov AS. Selective constraint in intergenic regions of human and mouse genomes. Trends Genet. 2001;17(7):373-6.

Ready to submit your research? Choose BMC and benefit from:

- fast, convenient online submission

- thorough peer review by experienced researchers in your field

- rapid publication on acceptance

- support for research data, including large and complex data types

- gold Open Access which fosters wider collaboration and increased citations

- maximum visibility for your research: over $100 \mathrm{M}$ website views per year

At BMC, research is always in progress.

Learn more biomedcentral.com/submissions 